\title{
Demonstrating the influence of UV rays on living things
}

\author{
Kouichi Morimoto \\ Nara University of Education, Japan
}

\begin{abstract}
Ultraviolet (UV) rays are used in our daily lives for a variety of purposes, including detection of counterfeit bank notes and passports, sterilisation in hospitals and water treatment plants, and in medical treatments for diseases such as athlete's foot. However, a majority of students are unaware of UV rays and their effects. This practical introduces students to the different types of UV rays and their effects on living things, using appropriate teaching materials and equipment. The effects of exposure to UV-B (fluorescent) and UV-C (germicidal) lamps are demonstrated using bananas, duckweed and the fruit fly. Students can observe photoreactivation when living samples are exposed to the UV-C followed by irradiation with the UV-B lamp.
\end{abstract}

Key words: UV rays, Photoreactivation, Banana, Duckweed, Fruit fly.

\section{Introduction}

Ultraviolet (UV) rays are utilised our daily life for various purposes. For example, they are used for detection for counterfeit bank notes and passports, sterilisation in hospitals, hair salons and water filtering plants, medical treatment for athlete's foot and tanning. Low intensity UV rays are also emitted from fluorescent lamps, televisions and computer monitors. Thus, today we are taking an increasing interest in UV rays as concern about UV rays grows - it is known that the Atlantic ozone is decreasing (Stolarki et al., 1986) and the phenomenon has called for research on the effect of UV radiation on living things (Kerr and Mcelroy, 1993).

Nevertheless, most students have little awareness of UV rays and their effects, although most students seem to have some experience of sunburn and tanning. Although the term 'ultraviolet ray' is familiar, the knowledge of their effects is limited (Morimoto, 1999). Therefore, it is necessary to teach more about UV rays, especially their effect on living things, including human beings. Inevitably, this requires the development of appropriate teaching materials and equipment.

Various teaching materials and equipment concerning the effect of UV radiation on living things have previously been reported, including E. coli (Nuttall, 1995), Vibrio natriegens (Delpech, 2001), and yeast (Mayo and Friedrichsen, 1993). However, the limitation of these materials is that they are microorganisms, which are often not familiar to students even in secondary school. It seems that more familiar materials - e.g. visible animals and plants - are required in the classroom setting. Also, UV radiation equipment should be simple so that any school laboratory can perform the experiments.

In this paper, such materials and equipment are introduced. The experiments use a banana, duckweed and fruit flies and a simple UV radiation device.

\section{Background knowledge and information}

Through this experiment students are expected to learn the effect of UV rays on living things. The experiments demonstrate various damage resulting from UV rays and the repair systems living organisms possess. In order to help students' understanding, maintain interest and explain the importance of learning about UV rays, it may be useful to provide students with some background knowledge and information in relation to the experiments presented in this paper. Such background information concerns the characteristics of the three types of UV rays, repair systems, exposure of human bodies to UV rays, and the effect of UV rays on plants. The following paragraphs suggest the information you could provide to students prior to demonstrating the experiments:

\section{The three types of UV rays}

Ultraviolet rays are mainly divided into three parts by wavelength - UV-C (200 - $280 \mathrm{~nm})$, UV-B (280 - $315 \mathrm{~nm})$ and UVA $(315-400 \mathrm{~nm})$. Though the sun emits all kinds of UV rays, only UV-A and UV-B rays reach the ground because the Earth's atmosphere and ozone layer absorbs UV-C wavelengths. It is known that UV-A causes the skin to tan and that UV-B causes sunburn. Although UV-C is prevented from reaching the Earth by the ozone layer, it is given out from welding arcs and also arises from the reflection of the sun from snow at high altitude. The depletion of the ozone layer has caused concern because of the implications of less protection from the sun's rays.

UV-C is known to be a strong mutagen of DNA. UV radiation with a wavelength of about $260 \mathrm{~nm}$ is absorbed strongly by bases of DNA and one consequence is the photochemical fusion of two adjacent pyrimidines into the cyclobutane thymine dimer (pyrimidine dimer) and 6-4 photoproduct (Watson et al., 1987). Recently, it was said that UV-B is also quite harmful to 
living things as it can induce the similar dimer. The formula $\mathrm{E}=\mathrm{h} v(1 / \lambda)[\mathrm{E}$ : energy; $\mathrm{h}$ : Planck constant; $\nu$ : frequency; $\lambda$ : wavelength], which calculates the energy of the UV rays, shows that the UV-B energy is lower than UV-C energy, and that damage by UV-B is little. However, UV-B deeply penetrates into the body of living things. The cornea of the eye is particularly susceptible to UV-C, whereas UV-B damages the lens which can lead to cataracts (Memmer 1989). UV-C and UV-B also cause skin cancer - UV-B damages the dermis, while UV-C damages the epidermis.

\section{Repair systems}

Though the DNA of skin cells exposed to normal sunlight acquires thousands of dimers per day, they are removed by repair systems. There are three kinds of repair system. The first is an excision repair system, which removes damaged bases or backbone segments of DNA. This excision repair system of dimers and other bulky lesion damage is initiated by the Uvr $\mathrm{ABC}$ endonuclease enzyme (Watson et al., 1987). This repair system can run in dark conditions and most living things have the system. The second repair system is recombinational. In this system, when all information is lost at the site of damage it can be recovered by taking a corresponding DNA segment from a separate identical DNA molecule (Watson et al., 1987).

The third repair system is called photoreactivation and is seen in some living things such as fruit flies and bananas. Photoreactivation is carried out by an enzyme called photolyase, which binds pyrimidine dimers, using visible light, changing the dimer ring back into individual pyrimidine bases.

Humans who do not have these repair systems are diagnosed with xeroderma pigmentosum, a rare heritable skin disease, and tend to contract skin cancer because their skin is abnormally sensitive to UV or sunlight. Duckweed is an example of a plant lacking a repair system and it is easily influenced and killed by UV rays. Therefore, it is often used as an environmental indicator.

\section{Exposure of human bodies to UV}

The extent of exposure of human bodies to UV rays varies according to lifestyle. According to Herlihy (1994), it is also determined by sporting activities - yachting causes the highest exposure and swimming the lowest. It is thought that the highly irradiated dose received during yachting is related to reflection from the water surface.

Osborne and Young (1998) report the extent of exposure to UV rays in relation to age and sex. They found that irrespective of sex, children less than five years old tend to be exposed more than other age groups. They also report that while men are highly exposed in their teens and 20 s, women are highly exposed in their 30 s due to taking their children out of doors. To reduce the influence of UV rays, it is effective to apply sunscreen to the skin.

\section{The effect of UV on plants}

UV rays damage plants in various ways, by decreasing chlorophylls and inhibiting growth and bud germination. It is known that when plants are exposed to various stresses, production of stress proteins is induced to protect against the stresses. UV-B induces the synthesis of flavonoids, which are found in epidermal vacuoles and function as filters by absorbing UV rays (Tenin et al., 1991).

\section{Materials and equipment}

As well as describing the materials used in this study, it's appropriate to explain why they are particularly suitable for use in secondary school laboratories.

\section{Banana}

A banana is inexpensive and widely available for purchase, so can therefore be prepared in any secondary school.

In the experiment, an unripe banana was used because the influence of UV rays is detected by peel colour change from yellow to brown. It is difficult to detect the influence of UV radiation on a mature banana, when peel already exhibits brown spots. Also, rather than several bananas, one banana divided into four sections was used, in order to compare different influences of UV rays clearly.

\section{Duckweed}

Duckweed is easily obtained in ponds during spring and summer, is familiar to many students and is therefore considered a useful material in school science. For example, Kawakami (1997) introduces duckweed as the indicator of the influence of coins (i.e. metals) on living things.

The species used in this study was Lemna paucicostata Hegelmaier. The culture solution was Murashige and Skooge (MS solution), whose components are shown in Kawakami et al. (1997). The duckweed was collected from a pond at Nara University of Education. Its frond size was about $5 \mathrm{~mm}$. Three individual duckweed fronds were used.

\section{Fruit flies}

Fruits flies (Drosophila melanogaster) are popular teaching materials in school and are used for various experiments. The flies can be purchased from educational institutes and teaching materials companies and also collected using a banana trap. Moreover, the existence of a lot of mutant strains of fruit fly is an advantage. If a strain of fly deficient in the UV damage repair system, or the photoreactivation system can be obtained, students will be able to do advanced experiments, which this study did not show. The species used in this study was Drosophila melanogaster Meigen, and the strain was Canton S (Wild type). The third instar larvae were used.

\section{UV radiation device}

The UV radiation device used in these experiments is shown in Figure 1. The device includes a normal fluorescent radiation device, a box and a blackout curtain. The UV-B lamp and UV$C$ lamp replaced the normal fluorescent lamp radiation devices - Toshiba health fluorescent lamp (FL20SE 20W) was used as UV-B irradiation; Toshiba germicidal lamp (GL10 10W) was used as UV-C irradiation.

The blackout curtain is used to prevent the leakage of UV rays. Moreover, to prevent operator exposure to UV radiation during the experiment, a special safely helmet and gloves were worn during the irradiation steps.

UV radiation dose $\left(\mathrm{J} / \mathrm{m}^{2} \mathrm{~s}\right.$ : Joule per square meter second $)$ is determined by the distance between the material and the lamp, and radiation time. Table 1 shows the distance and time that are required to deliver certain UV radiation doses. For example, to deliver $1000 \mathrm{~J} / \mathrm{m}^{2}$ rays by a UV-C lamp, it is required that the distance between a material and UV-C lamp is $22 \mathrm{~cm}$ and time 


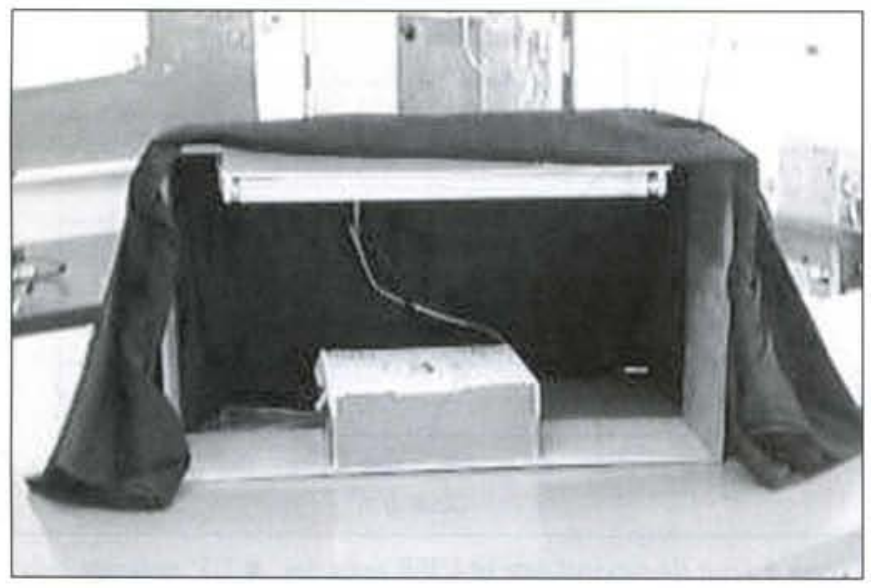

Figure 1 The UV radiation device.

is 224 seconds. The data shown in Table 1 are based on values measured by a UV dosimeter (UVR-2, Topkon Japan).

Thus, this study used a simple and safe device, which any secondary teachers can assemble and use easily.

Table 1. Radiation time and distance between materials and lamp for each dose of UV radiation.

\begin{tabular}{rcccc}
\hline & \multicolumn{2}{c}{ UV-B } & \multicolumn{2}{c}{ UV.C } \\
\hline Dose $\mathrm{J} / \mathrm{m}^{2}$ & Distance cm & Time sec & Distance cm & Time sec \\
\hline 100 & 26 & 23 & 33 & 33 \\
500 & 26 & 115 & 33 & 165 \\
1000 & 15 & 152 & 22 & 224 \\
1500 & 15 & 228 & 22 & 336 \\
2000 & 15 & 304 & 22 & 448 \\
\hline
\end{tabular}

\section{Methods}

\section{UV radiation of banana}

In order to perform the various treatments on one banana, the top quarter of the banana, including the stem, was covered by aluminium foil prior to any UV treatment and used as the control section. The second section was covered by aluminium foil after UV radiation, the third section had sunscreen (NeosunscreenN ANESSA Shiseido Japan) applied to it and was subsequently covered by aluminium foil after UV radiation. The lower quarter section was exposed to two fluorescent (UV-B) lamps at a distance of $14 \mathrm{~cm}$ for 24 hours at $25^{\circ} \mathrm{C}$ following UV radiation. This treatment is called photoreactivation treatment. UV treatment was delivered at a dose of $1000 \mathrm{~J} / \mathrm{m}^{2}$ (UV-C). The treated banana was then stored under dark conditions at room temperature for five days.

\section{UV radiation of duckweed}

Duckweed fronds were put in the culture medium (about $20 \mathrm{ml})$ in a glass petri dish $(9 \mathrm{~cm}$ diameter $)$ and UV radiation was carried out in the UV radiation device. UV radiation doses on separate samples were $0 \mathrm{~J} / \mathrm{m}^{2}$ (no radiation), $10 \mathrm{~kJ} / \mathrm{m}^{2}$ (UVB) and $2 \mathrm{k} \mathrm{J} / \mathrm{m}^{2}$ (UV-C). After UV irradiation, the duckweed was transferred to a $500 \mathrm{ml}$ beaker containing the culture medium. The number of fronds were measured according to Miura and Hirahara (1979). After each UV irradiation, cultures were left under a windowsill. The number of fronds was counted everyday at three o'clock in the afternoon. When the amount of cultured solution had reduced due to evaporation, distilled water was added to maintain the volume.

\section{$U V$ radiation of fruit fly}

A small amount of water was poured into a glass petri dish (9 $\mathrm{cm}$ diameter) and 10 third instar larvae were placed in it. UV irradiation was performed in the UV radiation device under experimental conditions. After UV radiation, the fruit flies were transferred to bottles containing standard medium. In the photoreactivation experiment, the UV-irradiated larvae were then exposed to two fluorescent (UV-B) lamps at distance of $14 \mathrm{~cm}$ for 24 hours at $25^{\circ} \mathrm{C}$. Both sets of larvae were then stored in the dark at $25^{\circ} \mathrm{C}$ until they reached maturity. The survival of UVirradiated larvae to adulthood was observed respectively every day. The experimental procedure is shown in Figure 2.

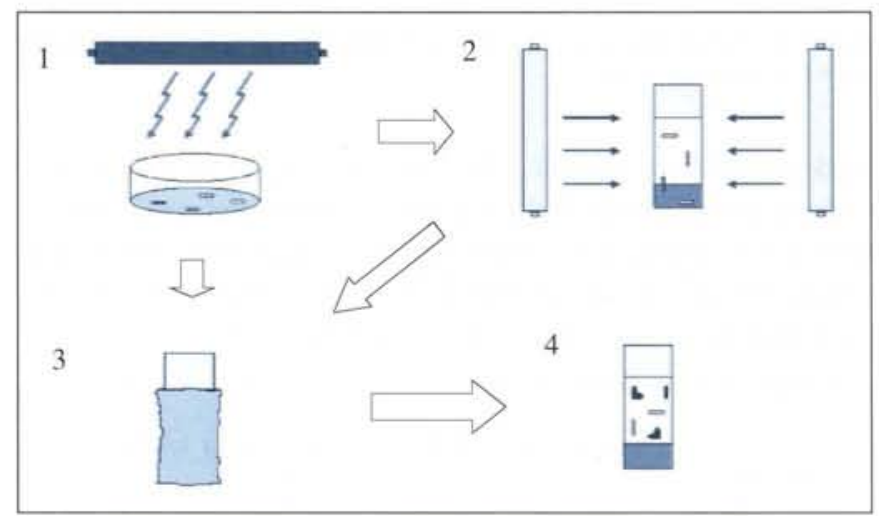

Figure 2 The UV radiation procedure. $1 \rightarrow 3 \rightarrow 4=U V$ radiation only; $1 \rightarrow 2 \rightarrow 3 \rightarrow 4=$ photoreactivation treatment.

\section{Results and discussion}

\section{Banana}

Figure 3 shows the results of banana on the fifth day following irradiation. The top quarter of the banana is yellow and dotted. As this section was not irradiated by any light, it is likely that the colour change is due to maturity. The colour of the next section is brown, and UV damage to the banana can be seen. It resembles a human suntan, and from this result, the students will understand the effect of UV radiation on living things. Only the banana peel is affected; the banana itself is not harmful for human consumption and is therefore edible. When the peel was cut vertically, the colour change was observed in only a few layers on the surface of the fruit. This shows that UV rays cannot penetrate the banana deeply. It is thought that the reason the banana peel changes colour is that the banana contains a lot of phenol compounds. The third section is a light brown colour and the protective nature of sunscreen to UV is clearly shown. The lower quarter section is also a light brown colour. This section was irradiated by the fluorescent lamp after UV irradiation and thus displays photoreactivation. It is clear that during the fluorescent lamp treatment the UV damage was repaired. Though the damage to this section did not recover completely, it can be seen that damage was certainly reduced. The result can demonstrate the photoreactivation phenomenon, which many students will not have been aware of.

\section{Duckweed}

The number of duckweed fronds counted during the experiment is shown in Figure 4. Duckweed under $0 \mathrm{~J} / \mathrm{m}^{2}$ (no radiation) increased its number of fronds gradually. The number on the 12 th day was 63 . In contrast, the number of UV-irradiated 


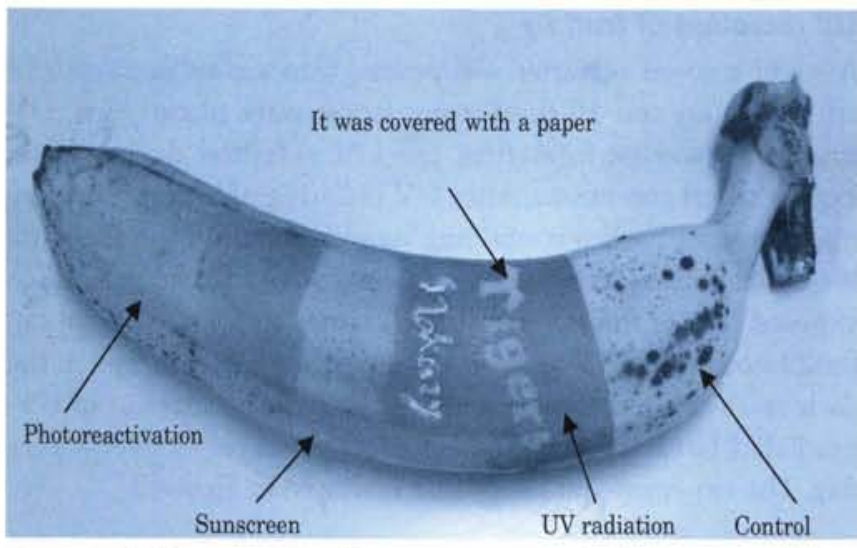

Figure 3 The banana, exposed to UV-C $1000 \mathrm{~J} / \mathrm{m}^{2}$, shown on the fifth day after UV radiation exposure.

duckweed fronds (UV-B 10k J/m2, 2k J/m2) increased only slightly. Some fronds lost their green colour and some turned brown. UV irradiation is likely to have induced this effect. The results of this experiment demonstrate the influence of UV rays on plants, especially plants without a repair system.

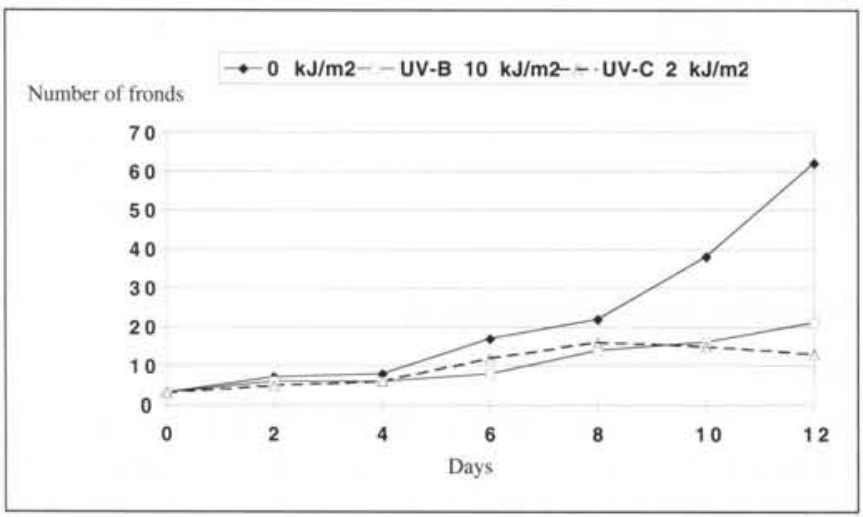

Figure 4 Change in number of duckweed fronds after UV radiation expo. sure. $\mathrm{O} \mathrm{J} / \mathrm{m}^{2} ; \square U V-B 10 \mathrm{KJ} / \mathrm{m}^{2} ; \Delta U V-\mathrm{C} 2 \mathrm{~K} \mathrm{~J} / \mathrm{m}^{2}$

\section{Fruit fly}

Figure 5 shows the result of UV-B irradiation on fruit flies. The survival rate is almost zero at $1000 \mathrm{~J} / \mathrm{m}^{2}$ under UV-B irradiation only. On the other hand, during photoreactivation treatment, the survival rate didn't decrease to zero until around $2400 \mathrm{~J} / \mathrm{m}^{2}$. Similarly, Figure 6 shows the result of fruit flies under UV-C irradiation. The survival rate is almost zero at $300 \mathrm{~J} / \mathrm{m}^{2}$ under UV-C irradiation only, but during photoreactivation treatment, the survival rate didn't decrease to zero until around $700 \mathrm{~J} / \mathrm{m}^{2}$. This demonstrates the effectiveness of photolyase in repairing UV damage to the fruit flies during the photoreactivation process. It is reported that pyrimidine dimers are the major cause of UV-induced lethal phenotypes in excisionless Drosophila larvae (Ryo and Kondo, 1986) and it's hoped that through this practical students can learn about the photoreactivation system of fruit flies.

Figure 7 shows the dead fruit fly that was observed using a microscope. The stage at death was pupa - this fruit fly could develop from larva to pupa, but it could not develop further to the adult stage. Black spots that resembled sunburn were observed and abnormal eye rudiment was observed in another dead pupa. It is known that excisionless larvae show a high incidence of somatic eye colour mutation after UV irradiation (Ryo
Survival rate

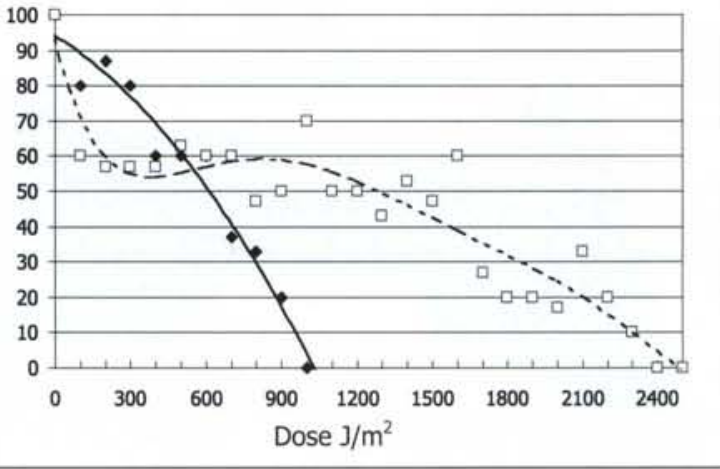

Figure 5 Fruit fly survival rate in UV-B radiation. $-U V$ radiation only; $\square$ Photoreactivation.

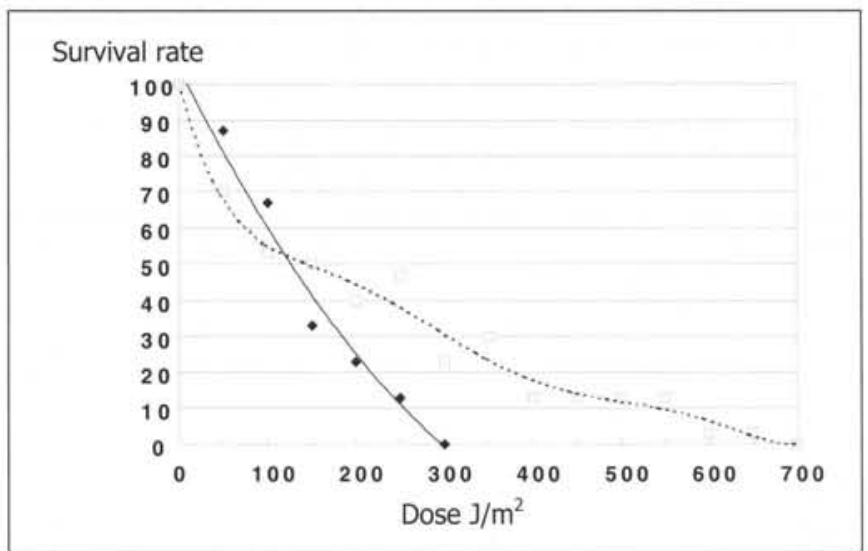

Figure 6 Fruit fly survival rate in UV-C radiation. $\bullet U V$ radiation only; $\square$ Photoreactivation.

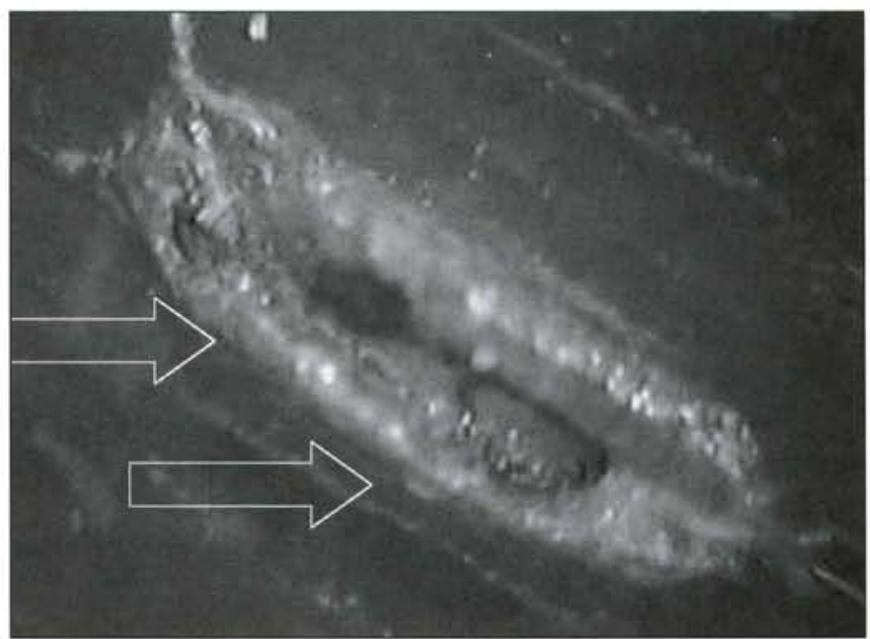

Figure 7 The dead body of fruit fly pupa, observed under microscope $(x 20)$.

and Kondo, 1986) and it is thought that the phenomenon observed in this experiment is similar to that documented. As the wild type fruit fly was used in this experiment, only a few dead bodies with abnormal eye rudiment were observed. Abnormal eye in adult fruit flies is induced by various mutagens. Therefore, in order to detect effects caused by some mutagen, observing the eye may be effective method.

\section{Conclusion}

This study attempted to develop teaching materials suitable for use in secondary schools, to demonstrate the effect of UV rays 
on living things. The experiments introduced in this paper use a banana, duckweed and fruit flies and a simple UV radiation device, which are simple, safe and easily obtainable. Additionally, the time required to obtain experiment results is relatively short. The experiments have been shown to demonstrate various phenomena concerning UV rays and help students' understanding of the effect of UV rays on living things.

There is scope for further more advanced experiments to subsequently be developed, and the experiments presented in this paper will hopefully inspire teachers in secondary school biology classes.

\section{References}

Delpech R (2001) Using Vibrio natriegens for studying bacterial population growth, artificial selection, and the effects of UV radiation and photo-reactivation. Joumal of Biological Education, 35, 93 - 97.

Herlihy E, Peter H G, Colin R R and Michael J (1994) Personal dosimetry of solar UV radiation for different outdoor activities. Photochemistry \& Photobiology, 60, $288-294$.

Kawakami S, Oda T and Ban R (1997) The influence of coins on the growth of duckweed. Joumal of Biological Education, 31, 116 - 118.

Kerr J B and Mcelroy C T (1993) Evidence for large upward trends of Ultraviolet-B radiation linked to Ozone depletion. Science, 262, 1032 -1034 .

Mayo L T and Friedrichsen P J (1993) Using yeast and ultraviolet radiation to introduce the scientific method. American Biology Teacher, $55,42-43$.

Memmer M K (1989) Preventing eye damage from the sun's Ultraviolet light: What health educators should teach. Heath education, 20, 42 47.

Miura, M and Hirahara, S (1979) Equilibrium and its breakdown in the mini-ecosystem (in Japanese). Live Teaching Materials Information Service, 53, $196-198$.

Morimoto K (1999) UV radiation awareness (in Japanese). The Bulletin of Japanese Curriculum Research and Development, 22, 19 - 29.

Nuttall D (1995) A simple biological indicator of solar ultraviolet radiation. Joumal of Biological Education, 29, 246 - 248.

Osborne J and Young A R (1998) The biological effects of ultraviolet radiation: a model for contemporary science education. Joumal of Biological Education, 33, 10 - 15.

Ryo $\mathrm{H}$ and Kondo S (1986) Photoreactivation rescue and hypermutability of ultraviolet irradiated excisionless Drosophila melanogaster larvae. Proceedings Natural Academic Science, 83, 3366 3370.

Stolarski R S, Krueger A J, Schoeberl M R, Mcpeters R D, Newman P A and Alpert J C (1986) Nimbus 7 satellite measurements of the springtime Antarctic ozone decrease. Nature, 322, 808 - 811.

Teveni M, Braun J and Fieser G (1991) The protective function of the epidermal layer of rye seedlings against ultraviolet-B radiation. Photochemical \& Photobiology, 53, $329-333$.

Watson J D, Hopkins N H, Roberts J W, Steitz J A and Weiner A M (1987) Molecular Biology of the Gene. San Francisco, USA: Benjamin Cummings.

\section{Appendix}

Shiseido Co., www.shiseido.co.jp

Topcom, Japan www.topcom.co.jp

Toshiba Corporation, Japan. www.toshiba.co.jp

Dr Kouchi Morimoto is Associate Professor at the Nara University of Education, Takabatakecho, Nara City, 630-8528, Japan. Tel: +81 74227 9205; Fax: +81742 27 9205; Email: morimoto@naraedu.ac.jp 